\title{
Generalized Version of Complementary Lindley Power Series Distribution with Application
}

Zahoor Ahmad

P.G Department of Statistics, University of Kashmir, Srinagar

zahoor151@gmail.com

Adil Rashid

P.G Department of Statistics, University of Kashmir, Srinagar

adilstat@gmail.com

T. R. Jan

P.G Department of Statistics, University of Kashmir, Srinagar

drtrjan@gmail.com

\begin{abstract}
In this paper, we shall introduce a new class of generalized complementary compound lifetime distributions which is obtained by compounding generalized Lindley distribution with power series distribution. This new family of continuous lifetime distributions so obtained will be called Complementary Generalized Lindley Power Series (CGLPS) distribution. The proposed class of distribution contains several lifetime distributions as its special cases that are very flexible to accommodate different types of data sets since the probability density function and hazard rate can take up different forms such as increasing, decreasing and upside down bathtub shapes which have been shown through graphs for some selected values of parameters and the potentiality of proposed class has been tested statistically by using it to model some real life data set.
\end{abstract}

Keywords: Generalized Lindley distribution, Power series distribution, Compounding and order statistics.

Mathematics Subject Classification: 60E05, 62F10

\section{Introduction}

Regarding the compounding of probability, the work has been done in this particular area since 1920. It is well known that Green wood and Yule (1920) established a relationship between Poisson distribution and a negative binomial distribution through by compounding mechanism by treating the parameter in Poisson distribution as gamma variate. The probability parameter in binomial distribution is constant; Skellam (1948) derived a probability distribution from the binomial distribution by treating $p$, the constant probability of successes as a beta variable between the sets of trails and this distribution which is commonly called beta binomial distribution proved to be efficient over binomial distribution when it comes to fit accidental data. Gurland (1957) showed that some compound distribution may also be called generalized distribution because there exists some interrelations between a certain class compound and generalized distributions. Sankaran (1970) proposed Poison Lindley distribution by allowing the parameter of Poisson distribution to vary according to Lindley distribution and this compound distribution was used for the analysis of count data sets but Sankaran didn't obtained MLE of unknown parameter $p$ due to computational difficulty. Kemp and Kemp (1966) obtained a compound of Poisson distribution with that of normal distribution by allowing the parameter of Poisson distribution as a normal variate, this particular distribution was called by them as Hermite distribution. Hermite distribution 
sometimes lacks any physical interpretation because the parameter in Poisson distribution is positive and the compounding distribution is supposed to vary according to that. However, if the normal compounding distribution has positive mean much larger than its variance then the probability of a negative value is almost negligible. Dubey (1970) obtained compound of gamma distribution by treating one of the parameter $\beta$ of gamma distribution as another gamma variate and reduced this compound distribution to the beta and $\mathrm{F}$ distribution by some suitable transformations. The problem of compounding of distribution was further addressed by Gerstenkorn $(1993,1996)$ who proposed several compound distributions, he obtained a compound of generalized gamma distribution with exponential distribution by treating one of the parameter of generalized gamma distribution as an exponential variate, he also obtained a compound of polya with beta distribution and the factorial moments of this particular compound distribution were also obtained with the help of sterling number of the $3^{\text {rd }}$ kind. Gertenkorn (2004) constructed another compound distribution by treating the probability parameter as a generalized beta variate in the extended version of negative binomial distribution. A new generalized negative binomial distribution was proposed by Gupta and Ong (2004), this distribution arises from Poisson distribution if the parameter $\beta$ follows generalized gamma distribution; the resulting distribution so obtained was applied to various data sets and can be used as better alternative to negative binomial distribution. Sometimes observable phenomenon under consideration does not start by taking a 'zero' value in that case zero truncated distribution provides better fit with this in mind, Ghitany Mutairi and Nadhrajah (2007) obtained a compound of zero truncated Poisson distribution with Lindley distribution, the estimation of parameters were also given by means of moments and MLE method. Zamani and Ismail (2010) constructed a new compound distribution by compounding negative binomial with one parameter Lindley distribution that provides good fit for count data where the probability at zero has a large value. Kumaraswamy distribution is a two parameter continuous probability distribution that has been obtained by Kumaraswamy (1980), this distribution is similar to the beta distribution but unlike beta distribution it has a closed form of cumulative distribution function which makes it very simple to deal with, Li, Haung and Zhao (2011) ascribed Kumaraswamy distribution to the probability parameter of binomial distribution and obtained and a new compound distribution called Kumaraswamy-binomial distribution which was used to model overdispersed binomial data. Adil and Jan (2013) obtained a compound of zero truncated GNBD with that of GBD, the new compound distribution so obtained has some desirable properties as it embodies several truncated compound distributions as special cases. Adil and Jan (2014a) treated the probability parameter of Geeta distribution as generalized beta variate the resulting distribution proved to be a general class for some discrete compound distributions. Based on same compounding mechanism Adil and Jan (2014b) treated the probability parameter in Consul distribution as random variable and ascribed a generalized beta distribution to it the resulting distribution was named as a compound of Consul with generalized beta distribution which embodies several compound distributions. Adil and Jan (2015a, 2016a) proposed two new competitive count data models, one is obtained by compounding Consul distribution with Kumaraswamy distribution that find its application in traffic analysis and other is obtained by compounding negative binomial distribution with Kumaraswamy distribution that finds its application in biological sciences. The potentiality of these two proposed models was tested by chi-square goodness of fit test by modeling the real world data sets from traffic, 
genetics and ecology. Pandey and Kumari (2016) obtained one parameter new lifetime distribution for modeling monotonic decreasing survival rates. Adil et al. (2017, 2018) obtained a versatile models that not only contains several sub-models as special cases but can be used to model various types of continuous lifetime data sets in parallel setting, they also obtained an advanced discrete count data model that finds its application in genetics when the data under consideration is overdispersed and heavy tailed.

The modeling of lifetime data has received prime attention of researchers from the last one decade. Many continuous probability models such as exponential, gamma, Weibull have been frequently used in statistical literature to analyze the lifetime data, but these probability models cannot be used efficiently to model the lifetime data that is bathtub shaped and have unimodal failure rates. To overcome this problem, researchers have focused their attention on compounding mechanism which helps in constructing suitable, flexible and alternative models to fit the lifetime data of different types.

Consider a system with $N$ components, where $N$, the number of components is being a discrete random variable with domain $N=1,2, \ldots$, The lifetime of $i^{\text {th }}$ component is a continuous random variable say $X_{i}$ that may follow any one of the lifetime distributions such as exponential, gamma, Weibull, Lindley etc. The suitable discrete distributions for $N$ may be geometric, zero truncated Poisson or power series distribution in general. The lifetime of such a system in series and parallel combination is defined and denoted by a non- negative random variable $Y=\min \left\{X_{i}\right\}_{i=1}^{N}$ or $Y=\max \left\{X_{i}\right\}_{i=1}^{N}$ respectively.

With this in mind Adamidis and Loukas (1998) constructed a two parameter lifetime distribution by compounding exponential distribution with geometric distribution called Exponential Geometric (EG) distribution. Tahmasbi and Rezaei (2008) obtained Exponential Logarithmic (EL) distribution by using the same compounding mechanism. Power series distribution contains several classical discrete distributions as its special cases, therefore Chahkandi and Ganjali (2009) introduced a compound class of Exponential Power Series (EPS) distribution which contains several compound distributions as its special cases. It is known that Weibull distribution contains exponential distribution as a special case, in view of this Morais and Baretto Souza (2011) replaced the exponential distribution with Weibull distribution in the compounding mechanism of EPS distribution and obtained a compound class of Weibull Power Series (WPS) distribution which contains EPS distribution as a special case. Recently one parameter Lindley distribution has been used frequently to model the lifetime data because it has been observed in several research papers that this distribution performs excellently well when it comes to fit the lifetime data. Adil and Jan (2016b) constructed a new family of lifetime distribution by compounding Lindley distribution with that of power series distribution that generalized LG distribution due to Zakerzadeh and Mahmoudi (2012). Furthermore, authors also discussed some special cases of the LPS family of compound distribution which are very flexible in terms of density and hazard rate functions. Applications and various mathematical properties such as moments, order statistics and parameter estimation through MLE of the proposed family of compound distribution were also discussed in detail. Adil and Jan (2018b) proposed a generalized version Lindley power series family of compound lifetime distributions which proved to be versatile because it not only generalizes most of the lifetime 
distributions but also have some attractive desirable properties in terms of hazard rate and density functions.

Here, in this particular paper we assume that there is no information about which factor was responsible for the component failure but only the maximum lifetime value among all risks is observed instead of the minimum lifetime value among all risks. The new distribution is a counterpart of the generalized Lindley power series distribution and therefore we call it complementary generalized Lindley power series (CGLPS) distribution.

The present paper is organized as follows: In section (2) we present the construction of the proposed family of lifetime distribution. Density, survival, hazard rate functions and some of the properties of the proposed family are given in section (3). Moment generating function of CGLPS class of distributions is given in section (4). Order statistics, their moments and parameter estimation are discussed in detail respectively in section (5) and (6). Special cases that include complementary Generalized Lindley Poisson, complementary Generalized Lindley logarithmic, complementary Generalized Lindley geometric and complementary Generalized Lindley binomial distributions are discussed section (7). Finally, real application and conclusion about new findings are respectively given in section (8) and (9)

\section{Construction of the Class}

Let $X_{1}, \ldots, X_{N}$ be an independent and identically distributed (iid) random variables following Lindley distribution with density function given by

$$
g(x ; \alpha, \beta)={\frac{\alpha \beta^{2}}{\beta+1}}^{2}(1+x)\left[1-\left(1+\frac{\beta x}{\beta+1}\right) e^{-\beta x}\right]^{\alpha-1} e^{-\beta x}
$$

Here, the index $N$ is itself a discrete random variable following zero truncated power series distribution with probability function given by

$$
P(N=n)=\frac{a_{n} \lambda^{n}}{C(\lambda)}, \quad n=1,2, \ldots
$$

where $a_{n}$ depends only on $n, C(\lambda)=\sum_{n=1}^{\infty} a_{n} \lambda^{n}$ and $\lambda>0$ is such that $C(\lambda)$ is finite. Below given Table 1 shows useful quantities of some zero truncated power series distributions such as Poisson, logarithmic, geometric and binomial (with $\mathrm{m}$ being the number of replicas) distributions.

\begin{tabular}{|l|l|l|l|l|l|l|}
\hline \multicolumn{7}{|c|}{ Table 1: Useful quantities of Some Power Series Distribution } \\
\hline Distribution & $a_{n}$ & $C(\lambda)$ & $C^{\prime}(\lambda)$ & $C^{\prime \prime}(\lambda)$ & $C^{-1}(\lambda)$ & $\lambda$ \\
\hline Poisson & $n !^{-1}$ & $e^{\lambda}-1$ & $e^{\lambda}$ & $e^{\lambda}$ & $\log (\lambda+1)$ & $\lambda \in(0, \infty)$ \\
\hline Logarithmic & $n^{-1}$ & $-\log (1-\lambda)$ & $(1-\lambda)^{-1}$ & $(1-\lambda)^{-2}$ & $1-e^{-\lambda}$ & $\lambda \in(0,1)$ \\
\hline Geometric & 1 & $\lambda(1-\lambda)^{-1}$ & $(1-\lambda)^{-2}$ & $2(1-\lambda)^{-3}$ & $\lambda(\lambda+1)^{-1}$ & $\lambda \in(0,1)$ \\
\hline Binomial & $\left(\begin{array}{l}m \\
n\end{array}\right)$ & $(\lambda+1)^{m}-1$ & $m(\lambda+1)^{m-1}$ & $\frac{m(m-1)}{(\lambda+1)^{2-m}}$ & $(\lambda-1)^{1 / m}-1$ & $\lambda \in(0, \infty)$ \\
\hline
\end{tabular}


Let $X_{(n)}=\max \left\{X_{i}\right\}_{i=1}^{N}$. The conditional cumulative distribution function of $X_{(n)} \mid N=n$ is given by

$$
\begin{aligned}
G_{X_{(n)} \mid N=n}(x) & =(G(x))^{n} \\
= & {\left[\left\{1-\left(1+\frac{\beta x}{\beta+1}\right) e^{-\beta x}\right\}^{\alpha}\right]^{n} }
\end{aligned}
$$

and

$$
P\left(X_{(n)} \leq x, N=n\right)=\frac{a_{n} \lambda^{n}}{C(\lambda)}\left[1-\left(1+\frac{\beta x}{\beta+1}\right) e^{-\beta x}\right]^{\alpha n}, x>0, n \geq 1 .
$$

The compound complementary Lindley power series family of distributions is defined by the marginal cumulative distribution function of $X_{(n)}$ :

$$
\begin{aligned}
F(x)=\sum_{n=1}^{\infty}\left[1-\left(1+\frac{\beta x}{\beta+1}\right) e^{-\beta x}\right]^{n} \frac{a_{n} \lambda^{n}}{C(\lambda)} & C\left[\lambda\left\{1-\left(1+\frac{\beta x}{\beta+1}\right) e^{-\beta x}\right\}^{\alpha}\right] \\
= & \frac{x(\lambda)}{C}, x>0
\end{aligned}
$$

From here, a random variable $X$ following complementary compound Lindley-power series distribution with parameters $\alpha, \beta$ and $\lambda$ will be denoted by $X \sim \operatorname{CGLPS}(\alpha, \beta, \lambda)$ This new class of distributions contains several distributions as its special cases. The complementary generalized Lindley geometric distribution and the complementary generalized Lindley Poisson distribution are obtained by taking $C(\lambda)=\lambda(1-\lambda)^{-1}$ with $\lambda \in(0,1)$ and $C(\lambda)=e^{\lambda}-1$ with $\lambda \in(0, \infty)$ respectively in (2). Similarly we obtain complementary generalized Lindley logarithmic distribution and complementary generalized Lindley binomial distribution by taking $C(\lambda)=-\log (1-\lambda) ., \lambda \in(0,1)$ and $C(\lambda)=(1+\lambda)^{-m}-1, \lambda \in(0, \infty)$ in (2) respectively. These cases will be discussed in detail in section (7)

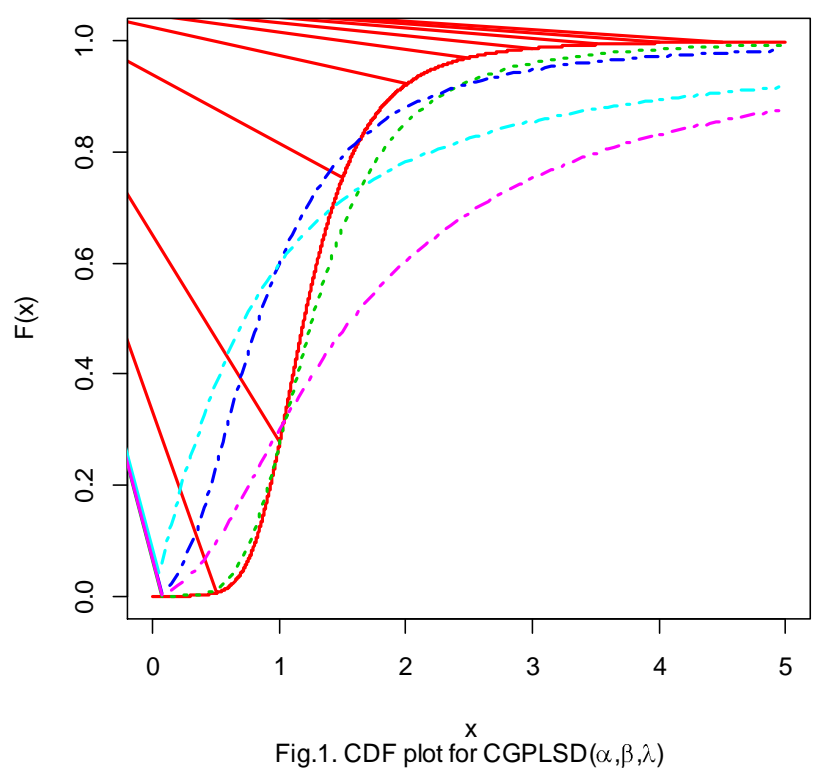




\section{Density, Survival and Hazard Rate Function}

The probability density function of complementary generalized Lindley power series distribution can be obtained by differentiating (2) both sides with respect to $x$.

$$
\begin{aligned}
& f(x)=\frac{d F(x)}{d x} \\
& f(x)=\frac{\beta^{2}}{C(\lambda)(\beta+1)} \alpha \lambda e^{-\beta x}(1+x)\left[1-\left(1+\frac{\beta x}{\beta+1}\right) e^{-\beta x}\right]^{\alpha-1} C^{\prime}\left[\lambda\left\{1-\left(1+\frac{\beta x}{\beta+1}\right) e^{-\beta x}\right\}^{\alpha}\right]
\end{aligned}
$$

$S(x)=1-F(x)$

$$
S(x)=1-\frac{C\left[\lambda\left\{1-\left(1+\frac{\beta x}{\beta+1}\right) e^{-\beta x}\right\}^{\alpha}\right]}{C(\lambda)}
$$

and the hazard function is

$$
\begin{aligned}
& h(x)=\frac{f(x)}{S(x)} \\
& h(x)=\frac{\frac{\beta^{2}}{\beta+1} \alpha \lambda e^{-\beta x}(1+x)\left[1-\left(1+\frac{\beta x}{\beta+1}\right) e^{-\beta x}\right]^{\alpha-1} C^{\prime}\left[\lambda\left\{1-\left(1+\frac{\beta x}{\beta+1}\right) e^{-\beta x}\right\}^{\alpha}\right]}{C(\lambda)-C\left[\lambda\left\{1-\left(1+\frac{\beta x}{\beta+1}\right) e^{-\beta x}\right\}^{\alpha}\right]}, x>0
\end{aligned}
$$

we will now study some properties of CGLPS distribution in the form of following important propositions.

Proposition 1: The generalized Lindley distribution is the limiting case of the CGLPS distribution when $\theta \rightarrow 0^{+}$.

Proof: From the cumulative distribution function of CGLPS distribution we have

$$
\lim _{\theta \rightarrow 0^{+}} F(x)=\lim _{\theta \rightarrow 0^{+}} \frac{C\left[\lambda\left\{1-\left(1+\frac{\beta x}{\beta+1}\right) e^{-\beta x}\right\}^{\alpha}\right]}{C(\lambda)}, x>0
$$

In view of the fact

$$
\begin{gathered}
C(\lambda)=\sum_{n=1}^{\infty} a_{n} \lambda^{n} \\
\lim _{\lambda \rightarrow 0^{+}} F(x)=\lim _{\lambda \rightarrow 0^{+}} \frac{\sum_{n=1}^{\infty} a_{n}\left[\lambda\left\{1-\left(1+\frac{\beta x}{\beta+1}\right) e^{-\beta x}\right\}^{\alpha}\right]^{n}}{\sum_{n=1}^{\infty} a_{n} \lambda^{n}}
\end{gathered}
$$

using the L' Hospital's rule, it follows 


$$
\begin{aligned}
& \lim _{\lambda \rightarrow 0^{+}} F(x)=\frac{a_{1}\left[1-\left(1+\frac{\beta x}{\beta+1}\right) e^{-\beta x}\right]^{\alpha}+\sum_{n=2}^{\infty} a_{n} n \lambda^{n-1}\left[1-\left(1+\frac{\beta x}{\beta+1}\right) e^{-\beta x}\right]^{\alpha n}}{a_{1}+\sum_{n=2}^{\infty} a_{n} n \lambda^{n-1}} \\
& \text { Hence we get that }=\left[1-\left(1+\frac{\beta x}{\beta+1}\right) e^{-\beta x}\right]^{\alpha} .
\end{aligned}
$$

which is the distribution function of generalized Lindley distribution.

Proposition 2: The densities of CGLPS class of distribution can be expressed as an infinite linear combination of densities of $n^{\text {th }}$ order statistics of Lindley distribution

$$
f(x)=\sum_{n=1}^{\infty} P(N=n) g_{n}(x, n)
$$

where $g_{n}(x, n)=\max \left(X_{1}, X_{2}, \ldots, X_{n}\right)$ is the $n^{\text {th }}$ order statistics of Lindley distribution

Proof: Using the fact that

$$
C^{\prime}(\lambda)=\sum_{n=1}^{\infty} n a_{n} \lambda^{n-1}
$$

The pdf of CGLPS distribution takes the form after using the above argument. Therefore it follows

$$
\begin{gathered}
f(x)=\frac{\beta^{2}}{\beta+1} \alpha(1+x) e^{-\beta x}\left[1-\left(1+\frac{\beta x}{\beta+1}\right) e^{-\beta x}\right]^{\alpha-1} \sum_{n=1}^{\infty} n \frac{a_{n} \lambda^{n}}{C(\lambda)}\left[1-\left(1+\frac{\beta x}{\beta+1}\right) e^{-\beta x}\right]^{n-1} \\
f(x)=\sum_{n=1}^{\infty} P(N=n) g_{n}(x, n)
\end{gathered}
$$

where $g_{n}(x, n)=\frac{n \beta^{2}}{\beta+1} \alpha(1+x) e^{-\beta x}\left[1-\left(1+\frac{\beta x}{\beta+1}\right) e^{-\beta x}\right]^{\alpha n-1}$

is the $n^{\text {th }}$ order statistics of generalized Lindley distribution. Therefore the densities of CGLPS distribution can be expressed as an infinite linear combination of the $n^{\text {th }}$ order statistics of generalized Lindley distribution. Hence it is obvious that properties of CGLPS distribution can be obtained from the $n^{\text {th }}$ order statistics $g_{n}(x, n)=\max \left(X_{1}, X_{2}, \ldots, X_{n}\right)$ of generalized Lindley distribution.

\section{Moment Generating Function}

The moment generating function of CGLPS distribution can be obtained from (4)

$$
M_{X}(t)=\sum_{n=1}^{\infty} P(N=n) M_{X_{(n)}}(t)
$$

where $M_{X_{(n)}}(t)$ is the moment generating function of $n^{\text {th }}$ order statistics of generalized Lindley distribution

$$
\begin{aligned}
M_{X_{(n)}}(t) & =\frac{n \alpha \beta^{2}}{\beta+1} \int_{0}^{\infty} e^{t x}(1+x) e^{-\beta x}\left[1-\left(1+\frac{\beta x}{\beta+1}\right) e^{-\beta x}\right]^{\alpha n-1} d x \\
& =\frac{n \alpha \beta^{2}}{\beta+1} \sum_{j=0}^{\infty}\left(\begin{array}{c}
\alpha n-1 \\
j
\end{array}\right) \sum_{k=0}^{\infty}\left(\begin{array}{c}
\alpha n-1-j \\
k
\end{array}\right)(-1)^{j}\left(\frac{\beta}{\beta+1}\right)^{\alpha n-j-k-1} \int_{0}^{\infty}(1+x) x^{\alpha n-j-k-1} e^{-[(\alpha n-j) \beta-t] x} d x
\end{aligned}
$$




$$
=\frac{n \alpha \beta^{2}}{\beta+1} \sum_{j=0}^{\infty}\left(\begin{array}{c}
\alpha n-1 \\
j
\end{array}\right) \sum_{k=0}^{\infty}\left(\begin{array}{c}
\alpha n-1-j \\
k
\end{array}\right)(-1)^{j}\left(\frac{\beta}{\beta+1}\right)^{\alpha n-j-k-1}\left\{\frac{\Gamma(\alpha n-j-k)}{((\alpha n-j) \beta-t)^{\alpha n-j-k}}+\frac{\Gamma(\alpha n-j-k+1)}{((\alpha n-j) \beta-t)^{\alpha n-j-k+1}}\right\}
$$

and it follows that

$$
\begin{aligned}
M_{X}(t)=\frac{\alpha \beta^{2}}{C(\lambda)(\beta+1)} \sum_{n=1}^{\infty} n a_{n} \lambda^{n} \sum_{j=0}^{\infty}\left(\begin{array}{c}
\alpha n-1 \\
j
\end{array}\right) \sum_{k=0}^{\infty}\left(\begin{array}{c}
\alpha n-1-j \\
k
\end{array}\right)(-1)^{j}\left(\frac{\beta}{\beta+1}\right)^{\alpha n-j-k-1} & \left\{\frac{\Gamma(\alpha n-j-k)}{((\alpha n-j) \beta-t)^{\alpha n-j-k}}+\frac{\Gamma(\alpha n-j-k+1)}{((\alpha n-j) \beta-t)^{\alpha n-j-k+1}}\right\}
\end{aligned}
$$

using the argument (4), the $k^{\text {th }}$ moment of CGLPS distribution about origin is

$$
\begin{aligned}
& E\left(X^{r}\right)=\sum_{n=1}^{\infty} P(N=n) \int_{0}^{\infty} x^{r} g_{n}(x) d x \\
& E\left(X^{r}\right)=\sum_{n=1}^{\infty} P(N=n) E\left(X_{(n)}{ }^{r}\right)
\end{aligned}
$$

Now consider

$$
\begin{aligned}
& E\left(X_{(n)}{ }^{r}\right)=\int_{0}^{\infty} x^{r} g_{n}(x) d x=\frac{n \alpha \beta^{2}}{\beta+1} \int_{0}^{\infty} x^{r}(1+x) e^{-\beta x}\left[1-\left(1+\frac{\beta x}{\beta+1}\right) e^{-\beta x}\right]^{\alpha n-1} d x \\
& =\frac{n \alpha \beta^{2}}{\beta+1} \sum_{j=0}^{\infty}\left(\begin{array}{c}
\alpha n-1 \\
j
\end{array}\right) \sum_{k=0}^{\infty}\left(\begin{array}{c}
\alpha n-1-j \\
k
\end{array}\right)(-1)^{j}\left(\frac{\beta}{\beta+1}\right)^{\alpha n-j-k-1} \int_{0}^{\infty}(1+x) x^{\alpha n-j-k+r-1} e^{-[(\alpha n-j) \beta] x} d x \\
& =\frac{n \alpha \beta^{2}}{\beta+1} \sum_{j=0}^{\infty}\left(\begin{array}{c}
\alpha n-1 \\
j
\end{array}\right) \sum_{k=0}^{\infty}\left(\begin{array}{c}
\alpha n-1-j \\
k
\end{array}\right)(-1)^{j}\left(\frac{\beta}{\beta+1}\right)^{\alpha n-j-k-1}\left\{\frac{\Gamma(\alpha n-j-k+r)}{((\alpha n-j) \beta)^{\alpha n-j-k+r}}+\frac{\Gamma(\alpha n-j-k+r+1)}{((\alpha n-j) \beta)^{\alpha n-j-k+r+1}}\right\}
\end{aligned}
$$

Hence we get

$$
\begin{aligned}
E\left(X^{r}\right)=\frac{\alpha \beta^{2}}{C(\lambda)(\beta+1)} \sum_{n=1}^{\infty} n a_{n} \lambda^{n} \sum_{j=0}^{\infty}\left(\begin{array}{c}
\alpha n-1 \\
j
\end{array}\right) \sum_{k=0}^{\infty}\left(\begin{array}{c}
\alpha n-1-j \\
k
\end{array}\right)(-1)^{j}\left(\frac{\beta}{\beta+1}\right)^{\alpha n-j-k-1} & \\
& \left\{\frac{\Gamma(\alpha n-j-k+r)}{((\alpha n-j) \beta)^{\alpha n-j-k+r}}+\frac{\Gamma(\alpha n-j-k+r+1)}{((\alpha n-j) \beta)^{\alpha n-j-k+r+1}}\right\} \text { (5) }
\end{aligned}
$$

\section{Order Statistics and Their Moments}

Order statistics are most oftenly used in statistics theory and practice. Order statistics are considered as one of the most fundamental tools in non-parametric and inferential statistics because they play an important role in quality control testing and reliability where a practitioner needs to predict the failure of future items based on the times of a few early failures.

Let $X_{1}, X_{2}, \ldots, X_{n}$ be a random sample from CGLPS distribution and $X_{1: n}, X_{2: n}, \ldots, X_{1: n}$ denote the corresponding order statistics. The pdf of $i^{t h}$ order statistics say $X_{i: n}$ is given by

$$
\begin{aligned}
f_{i: n}(x) & =\frac{n !}{(n-i) !(i-1) !}[F(x)]^{i-1}[1-F(x)]^{n-i} f(x) \\
& \left.=\frac{n ! f(x)}{(n-i) !(i-1) !}\left[\frac{C\left[\lambda\left\{1-\left(1+\frac{\beta x}{\beta+1}\right) e^{-\beta x}\right\}\right]}{C(\lambda)}\right]\right]^{\alpha}\left[1-\frac{C\left[\lambda\left\{1-\left(1+\frac{\beta x}{\beta+1}\right) e^{-\beta x}\right\}^{\alpha}\right]}{C(\lambda)}\right]^{n-i}
\end{aligned}
$$


Expression (6) can be equivalently written as

$$
f_{i: n}(x)=\frac{n !}{(n-i) !(i-1) !} \sum_{k=0}^{n-i}\left(\begin{array}{c}
n-i \\
k
\end{array}\right)(-1)^{k} f(x)[F(x)]^{k+i-1}
$$

Or

$$
f_{i: n}(x)=\frac{n !}{(n-i) !(i-1) !} \sum_{k=0}^{i-1}\left(\begin{array}{c}
i-1 \\
k
\end{array}\right)(-1)^{k} f(x)[1-F(x)]^{k+n-i}
$$

In view of the fact

$$
f(x)[F(x)]^{k+i-1}=\left(\frac{1}{k+i}\right) \frac{d}{d x}[F(x)]^{k+i}
$$

The associated CDF of $f_{i: n}(x)$ denoted by $F_{i: n}(x)$ becomes

$$
F_{i: n}(x)=\frac{n !}{(n-i) !(i-1) !} \sum_{k=0}^{n-i} \frac{\left(\begin{array}{c}
n-i \\
k
\end{array}\right)(-1)^{k}}{(k+i)}\left[\frac{C\left[\lambda\left\{1-\left(1+\frac{\beta x}{\beta+1}\right) e^{-\beta x}\right\}^{\alpha}\right]}{C(\lambda)}\right]^{k+i}
$$

Alternatively expression (7) can be written as

$$
F_{i: n}(x)=1-\frac{n !}{(n-i) !(i-1) !} \sum_{k=0}^{i-1} \frac{\left(\begin{array}{c}
i-1 \\
k
\end{array}\right)(-1)^{k}}{(k+n-i+1)}\left[1-\frac{C\left[\lambda\left\{1-\left(1+\frac{\beta x}{\beta+1}\right) e^{-\beta x}\right\}^{\alpha}\right]}{C(\lambda)}\right]^{k+n-i+1}
$$

Expression for $r^{\text {th }}$ moment of $i^{\text {th }}$ order statistics $X_{1: n, \ldots . .} X_{n: n}$ with CDF (7) can be obtained by using a well-known result due to Barakat and Abdelkadir (2004) as follows

$$
E\left(X_{i: n}^{r}\right)=r \sum_{k=n-i+1}^{n}(-1)^{k-n+i-1}\left(\begin{array}{l}
k-1 \\
n-i
\end{array}\right)\left(\begin{array}{l}
n \\
k
\end{array}\right) \int_{0}^{\infty} x^{r-1} S(x)^{k} d x
$$

where $S(x)$ is the survival function of CGLPS distribution. Therefore we have

$$
E\left(X_{i: n}^{r}\right)=r \sum_{k=n-i+1}^{n}(-1)^{k-n+i-1}\left(\begin{array}{l}
k-1 \\
n-i
\end{array}\right)\left(\begin{array}{l}
n \\
k
\end{array}\right) \int_{0}^{\infty} x^{r-1}\left(1-\frac{C\left[\lambda\left\{1-\left(1+\frac{\beta x}{\beta+1}\right) e^{-\beta x}\right\}^{\alpha}\right]}{C(\lambda)}\right)^{k} d x
$$

where $r=1,2,3 \ldots$ and $i=1,2, \ldots, n$

\section{Parameter Estimation}

Let $X_{1}, \ldots, X_{N}$ be a random sample with observed values $x_{1}, \ldots x_{n}$ from $\operatorname{CGLPS}(\lambda, \theta)$ distribution and let $\Theta=(\lambda, \theta)^{T}$ be the unknown parameter vector in the rest of the paper. The log-likelihood function is given by 


$$
\begin{aligned}
l_{n}=l_{n}(x, \Theta)= & 2 n \log \beta-n \log (\beta+1)+n \log \alpha+n \log \lambda-\beta \sum_{i=1}^{n} x_{i}+\sum_{i=1}^{n} \log \left(1+x_{i}\right)+ \\
& n(\alpha-1) \sum_{i=1}^{n} \log \left[1-\left(1+\frac{\beta x_{i}}{\beta+1}\right) e^{-\beta x_{i}}\right]-n \log C(\lambda)+\sum_{i=1}^{n} \log \left[C^{\prime}\left\{\lambda\left\{1-\left(1+\frac{\beta x_{i}}{\beta+1}\right) e^{-\beta x_{i}}\right\}\right\}\right]
\end{aligned}
$$

The corresponding score functions are

$$
\begin{gathered}
\frac{\partial l_{n}}{\partial \alpha}=\frac{n}{\alpha}+n \sum_{i=1}^{n} \log \left[1-\left(1+\frac{\beta x_{i}}{\beta+1}\right) e^{-\beta x_{i}}\right]+\lambda \sum_{i=1}^{n} \frac{\left\{1-\left(1+\frac{\beta x_{i}}{\beta+1}\right) e^{-\beta x_{i}}\right\}^{\alpha} \log \left\{1-\left(1+\frac{\beta x_{i}}{\beta+1}\right) e^{-\beta x_{i}}\right\}}{C^{\prime}\left\{\lambda\left\{1-\left(1+\frac{\beta x_{i}}{\beta+1}\right) e^{-\beta x_{i}}\right\}^{\alpha}\right\}} \\
\frac{\partial l_{n}}{\partial \beta}=\frac{2 n}{\beta}-\sum_{i=1}^{n} x_{i}-\frac{n}{\beta+1}+\sum_{i=1}^{n} \frac{C^{\prime \prime}\left[\lambda\left\{1-\left(1+\frac{\beta x_{i}}{\beta+1}\right) e^{-\beta x_{i}}\right\}^{\alpha}\right]}{C^{\prime}\left[\lambda\left\{1-\left(1+\frac{\beta x_{i}}{\beta+1}\right) e^{-\beta x_{i}}\right\}^{\alpha}\right]} \frac{\partial}{\partial \beta} \lambda\left\{1-\left(1+\frac{\beta x_{i}}{\beta+1}\right) e^{-\beta x_{i}}\right\}^{\alpha} \\
\frac{\partial l_{n}}{\partial \lambda}=\frac{n}{\lambda}-\frac{n C^{\prime}(\lambda)}{C(\lambda)}+\sum_{i=1}^{n} \frac{C^{\prime \prime}\left[\lambda\left\{1-\left(1+\frac{\beta x_{i}}{\beta+1}\right) e^{-\beta x_{i}}\right\}^{\alpha}\right]}{C^{\prime}\left[\lambda\left\{1-\left(1+\frac{\beta x_{i}}{\beta+1}\right) e^{-\beta x_{i}}\right\}^{\alpha}\right]}\left\{1-\left(1+\frac{\beta x_{i}}{\beta+1}\right) e^{-\beta x_{i}}\right\}^{\alpha}
\end{gathered}
$$

The maximum likelihood estimate of $\Theta$ say $\hat{\Theta}$ is obtained by solving the non-linear system of equations $U_{n}(\Theta)=\left(\frac{\partial l_{n}}{\partial \beta}, \frac{\partial l_{n}}{\partial \alpha}, \frac{\partial l_{n}}{\partial \lambda}\right)^{T}=0$. The solution of this non-linear system of equation can be found numerically by using software such as $\mathrm{R}$.

\section{Consequences of proposed model}

In this section we will study in detail some special sub-models of CGLPS distributions further more we also present expression for cdf, pdf, hazard rate and survival function. The graphical nature for different values of parameters is also studied to show the flexibility of these sub models.

\subsection{Complementary GeneralizedLindley Poisson Distribution (CGLPD)}

Poisson distribution is a special case of power series distribution for $C(\lambda)=e^{\lambda}-1$ and $C^{\prime}(\lambda)=e^{\lambda}$. Therefore cdf and pdf of a compound of Generalized Lindley Poisson (CGLP) distribution is obtained by using the same arguments in (2).

$$
F(x)=\frac{e^{\lambda\left[1-\left(1+\frac{\beta x}{\beta+1}\right) e^{-\beta x}\right]^{\alpha}}-1}{e^{\lambda}-1}, x>0 .
$$

The associated pdf, hazard and survival functions are respectively given by 


$$
\begin{gathered}
f(x)=\frac{\beta^{2}}{\beta+1} \lambda e^{-\beta x}(1+x) \frac{C^{\prime}\left[\left\{1-\left(1+\frac{\beta x}{\beta+1}\right) e^{-\beta x}\right\} \lambda\right]}{C(\lambda)} \\
f(x)=\frac{\beta^{2}}{\beta+1} \lambda(1+x) e^{-\beta x} e^{\lambda\left[1-\left(1+\frac{\beta x}{\beta+1}\right) e^{-\beta x}\right]}\left(e^{\lambda}-1\right)^{-1} \\
S(x)=1-\frac{e^{\lambda\left[1-\left(1+\frac{\beta x}{\beta+1}\right) e^{-\beta x}\right]^{\alpha}}-1}{e^{\lambda}-1} \text { and } h(x)=\frac{\beta^{2} \lambda(1+x) e^{-\beta x} e^{\lambda\left[1-\left(1+\frac{\beta x}{\beta+1}\right) e^{-\beta x}\right]^{\alpha}}}{(\beta+1)\left[e^{\lambda}-e^{\lambda\left\{1-\left(1+\frac{\beta x}{\beta+1}\right) e^{-\beta x}\right\}^{\alpha}}\right]}
\end{gathered}
$$

for $x, \beta>0,0<\lambda<\infty$ respectively. The expression for the $k^{\text {th }}$ moment of a random variable following CGLP distribution becomes by taking $a_{n}=n !^{-1}$ and $C(\beta)=e^{\lambda}-1$ in (5)
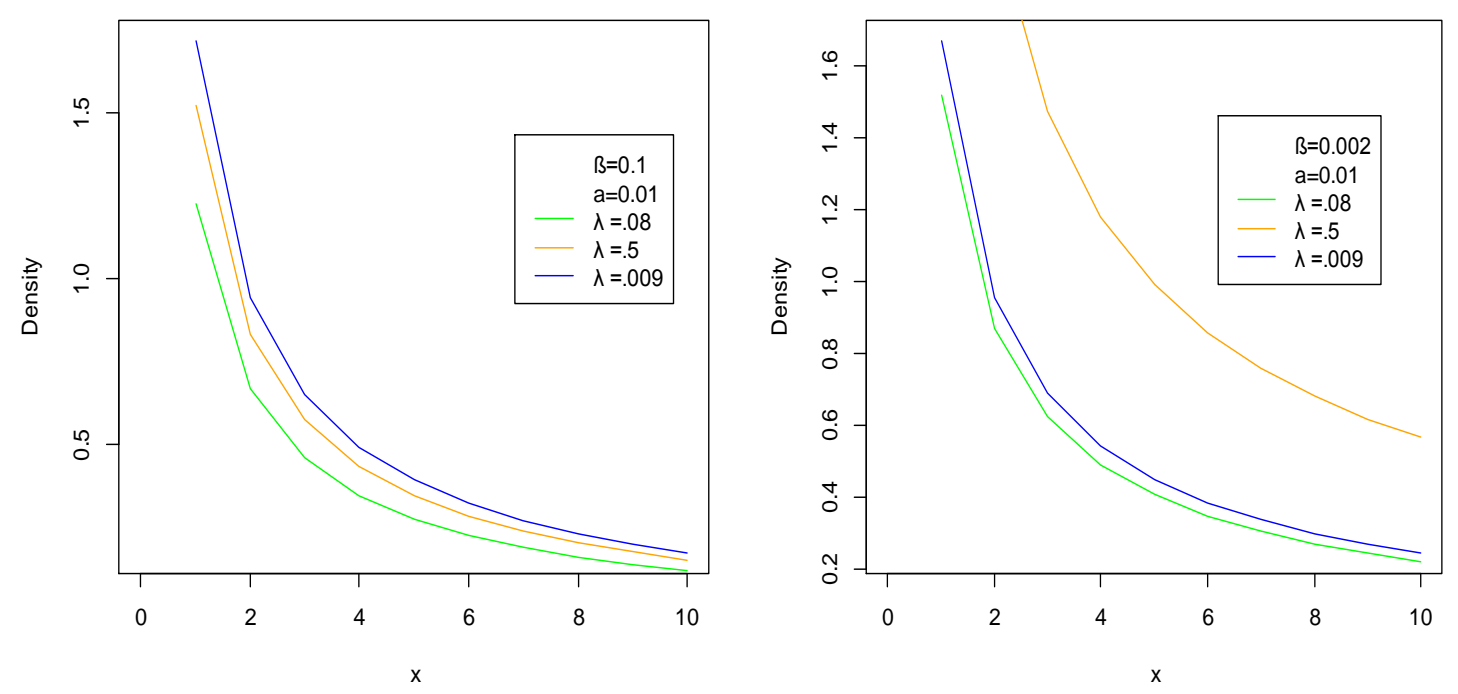

Fig .2: These graphs show the flexibilty of density of CGLPD for some selected values of parameters

\subsection{Complementary Generalized Lindley Logarithmic Distribution (CGLLD)}

Logarithmic distribution is a special case of PSD when $C(\lambda)=-\log (1-\lambda)$ and $C^{\prime}(\lambda)=(1-\lambda)^{-1}$. Therefore a compound of Complementary Generalized Lindley Logarithmic (CGLL) distribution is followed from (2) when we put $C(\lambda)=-\log (1-\lambda)$ in it.

$$
F(x)=\frac{\log \left[1-\lambda\left\{1-\left(1+\frac{\beta x}{\beta+1}\right) e^{-\beta x}\right\}^{\alpha}\right]}{\log (1-\lambda)}, x>0
$$

and the associated p.d.f, hazard rate and survival function are

$$
f(x)=\frac{\frac{\beta^{2}}{\beta+1} \lambda e^{-\beta x}(1+x)\left\{1-\left(1+\frac{\beta x}{\beta+1}\right) e^{-\beta x}\right\}^{\alpha-1}}{\left[\left\{\lambda\left\{1-\left(1+\frac{\beta x}{\beta+1}\right) e^{-\beta x}\right\}^{\alpha}\right\}-1\right][\log (1-\lambda)]}
$$




$$
\begin{gathered}
S(x)=1-\frac{\log \left[1-\lambda\left\{1-\left(1+\frac{\beta x}{\beta+1}\right) e^{-\beta x}\right\}^{\alpha}\right]}{\log (1-\lambda)} \\
h(x)=\frac{\frac{\beta^{2}}{\beta+1} \lambda(1+x) e^{-\beta x}\left\{1-\left(1+\frac{\beta x}{\beta+1}\right) e^{-\beta x}\right\}^{\alpha-1}}{\left[\left\{\lambda\left\{1-\left(1+\frac{\beta x}{\beta+1}\right) e^{-\beta x}\right\}^{\alpha}\right\}-1\right]\left[\log (1-\lambda)-\log \left\{1-\lambda\left\{1-\left(1+\frac{\beta x}{\beta+1}\right) e^{-\beta x}\right\}^{\alpha}\right\}\right]}
\end{gathered}
$$

for $x, \lambda>0$ and $0<\theta<1$ respectively.
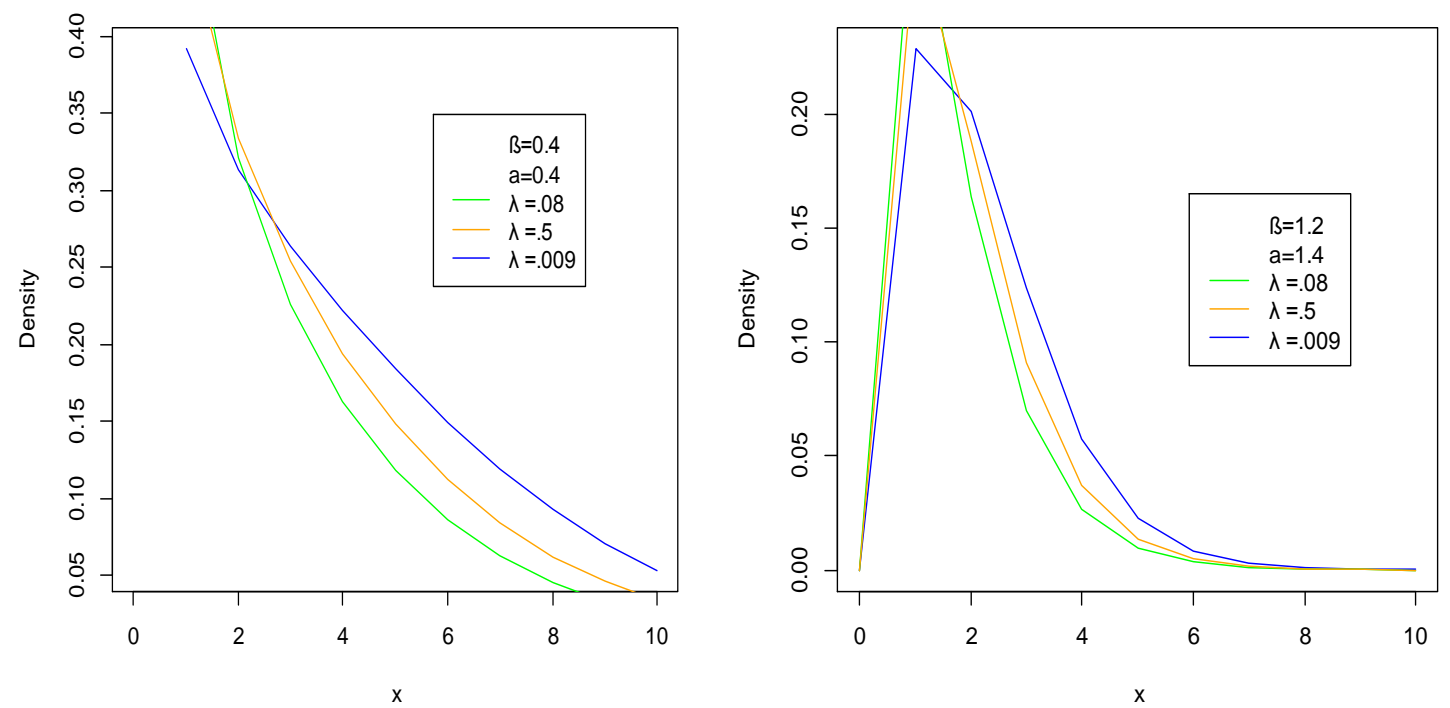

Fig .3: These graphs show the flexibilty of density of CGLLD for some selected values of parameters

The expression for the $k^{\text {th }}$ moment of a random variable following CGLL distribution becomes by taking $a_{n}=n^{-1}$ and $C(\lambda)=-\log (1-\lambda)$ in (5)

\subsection{Complementary Generalized Lindley Geometric Distribution (CGLGD)}

Geometric distribution is a particular case of PSD when $C(\lambda)=\lambda(1-\lambda)^{-1}$ and $C^{\prime}(\lambda)=(1-\lambda)^{-2}$. Therefore a compound of Complementary Generalized Lindley Geometric (CGLG) distribution is followed from (2) after using $C(\lambda)=\lambda(1-\lambda)^{-1}$ in it.

$$
F(x)=\frac{\left[\left\{1-\left(1+\frac{\beta x}{\beta+1}\right) e^{-\beta x}\right\}^{\alpha}\right](1-\lambda)}{\left[1-\lambda\left\{1-\left(1+\frac{\beta x}{\beta+1}\right) e^{-\beta x}\right\}^{\alpha}\right]}, x>0
$$

The associated pdf is

$$
f(x)=\frac{\alpha \beta^{2}}{\beta+1}(1-\lambda)(1+x) e^{-\beta x}\left[1-\left(1+\frac{\beta x}{\beta+1}\right) e^{-\beta x}\right]^{\alpha-1}\left[1-\lambda\left\{1-\left(1+\frac{\beta x}{\beta+1}\right) e^{-\beta x}\right\}^{\alpha}\right]^{-2}
$$




$$
\begin{aligned}
& S(x)=1-\frac{\left[\left\{1-\left(1+\frac{\beta x}{\beta+1}\right) e^{-\beta x}\right\}^{\alpha}\right](1-\lambda)}{\left[1-\lambda\left\{1-\left(1+\frac{\beta x}{\beta+1}\right) e^{-\beta x}\right\}^{\alpha}\right]} \\
& h(x)=\frac{\frac{\alpha \beta^{2}}{\beta+1}(1-\lambda)(1+x) e^{-\beta x}\left[1-\left(1+\frac{\beta x}{\beta+1}\right) e^{-\beta x}\right]^{\alpha-1}\left[1-\lambda\left\{1-\left(1+\frac{\beta x}{\beta+1}\right) e^{-\beta x}\right\}^{\alpha}\right]^{-1}}{1-\left[1-\left(1+\frac{\beta x}{\beta+1}\right) e^{-\beta x}\right]^{\alpha}}
\end{aligned}
$$

for $x, \beta, 0<\lambda<1$ respectively.
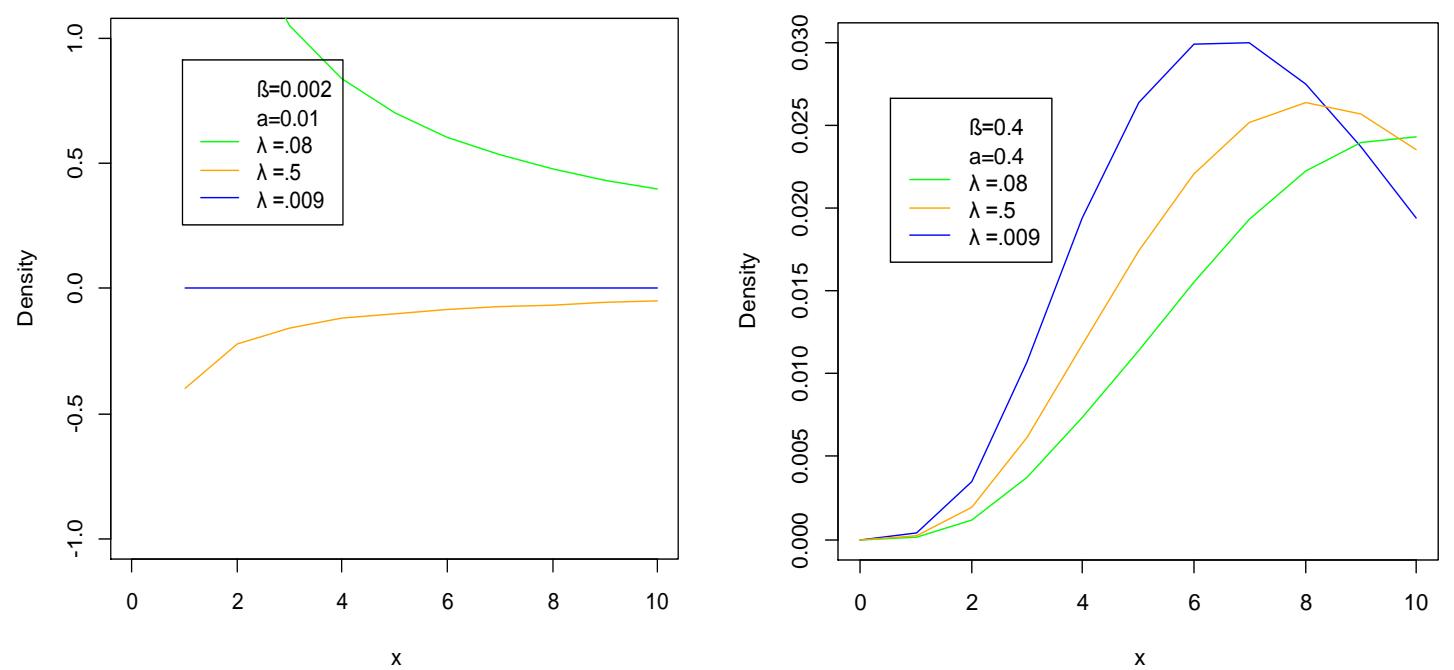

Fig .4: These graphs show the flexibilty of density of CGLGD for some selected values of parameters

\subsection{Complementary Generalized Lindley Binomial Distribution (CGLBD)}

Binomial distribution is a particular case of PSD for $C(\lambda)=(\lambda+1)^{m}-1$ and a compound of compound Complementary Generalized Lindley Binomial (CGLB) distribution is followed from (2) by using $C(\lambda)=(\lambda+1)^{m}-1$

$$
F(x)=\frac{\left[\lambda\left\{1-\left(1+\frac{\beta x}{\beta+1}\right) e^{-\beta x}\right\}^{\alpha}+1\right]^{m}-1}{(\lambda+1)^{m}-1}, x>0
$$

where $m$ is the positve integer. The associated pdf, hazard rate and survival functions are

$$
f(x)=\frac{\beta^{2}}{\beta+1} \alpha \lambda e^{-\beta x}(1+x)\left\{1-\left(1+\frac{\beta x}{\beta+1}\right) e^{-\beta x}\right\}^{\alpha-1} m\left[\lambda\left\{1-\left(1+\frac{\beta x}{\beta+1}\right) e^{-\beta x}\right\}^{\alpha}+1\right]^{m-1}
$$




$$
\begin{gathered}
S(x)=1-\frac{\left[\lambda\left\{1-\left(1+\frac{\beta x}{\beta+1}\right) e^{-\beta x}\right\}^{\alpha}+1\right]^{m}-1}{(\lambda+1)^{m}-1} \\
h(x)=\frac{\frac{\beta^{2}}{\beta+1} \alpha \lambda e^{-\beta x}(1+x)\left\{1-\left(1+\frac{\beta x}{\beta+1}\right) e^{-\beta x}\right\}^{\alpha-1} m\left[\lambda\left\{1-\left(1+\frac{\beta x}{\beta+1}\right) e^{-\beta x}\right\}^{\alpha}+1\right]^{m-1}}{1-\frac{\left[\lambda\left\{1-\left(1+\frac{\beta x}{\beta+1}\right) e^{-\beta x}\right\}^{\alpha}+1\right]^{m}-1}{(\lambda+1)^{m}-1}}
\end{gathered}
$$

respectively for $x, \beta>0,0<\lambda<\infty$. The expression for the $k^{\text {th }}$ moment of a random variable following CGLB distribution becomes by taking $a_{n}=\left(\begin{array}{l}m \\ n\end{array}\right)$ and $C(\lambda)=(\lambda+1)^{m}-1$ in (5). It may be noted here that these sub models are new lifetime distributions that have been obtained on specific parameter setting in CGLPSD.

\section{Application}

We consider a data set from Murthy et al. (2004) that gives failure times of 20 mechanical components. The data are: 0.067, 0.068, 0.076, 0.081, 0.084, 0.085, 0.085, $0.086,0.089,0.098,0.098,0.114,0.114,0.115,0.121,0.125,0.131,0.149,0.160,0.485$ Our aim is to fit this data by the proposed family of compound distributions. The MLE of unknown parameters and Akaike information criterion (AIC) and Bayesian information criterion (BIC) of the fitted distribution is given in table (3)

\section{Table 3: Analysis of model fitting}

$\begin{array}{cccc}\text { MODEL } & M L E & A I C & B I C \\ \text { CGLG } & \hat{\beta}=5.17, \hat{\lambda}=2.3, \hat{\alpha}=1 & -33.9 & -30.9 \\ \text { CGLP } & \hat{\beta}=30.3, \hat{\lambda}=16, \hat{\alpha}=1 & -61.1 & -58.1 \\ \text { CGLL } & \hat{\beta}=18.3, \hat{\lambda}=0.9, \hat{\alpha}=1 & -44.5 & -41.4\end{array}$


Histogram of $x$

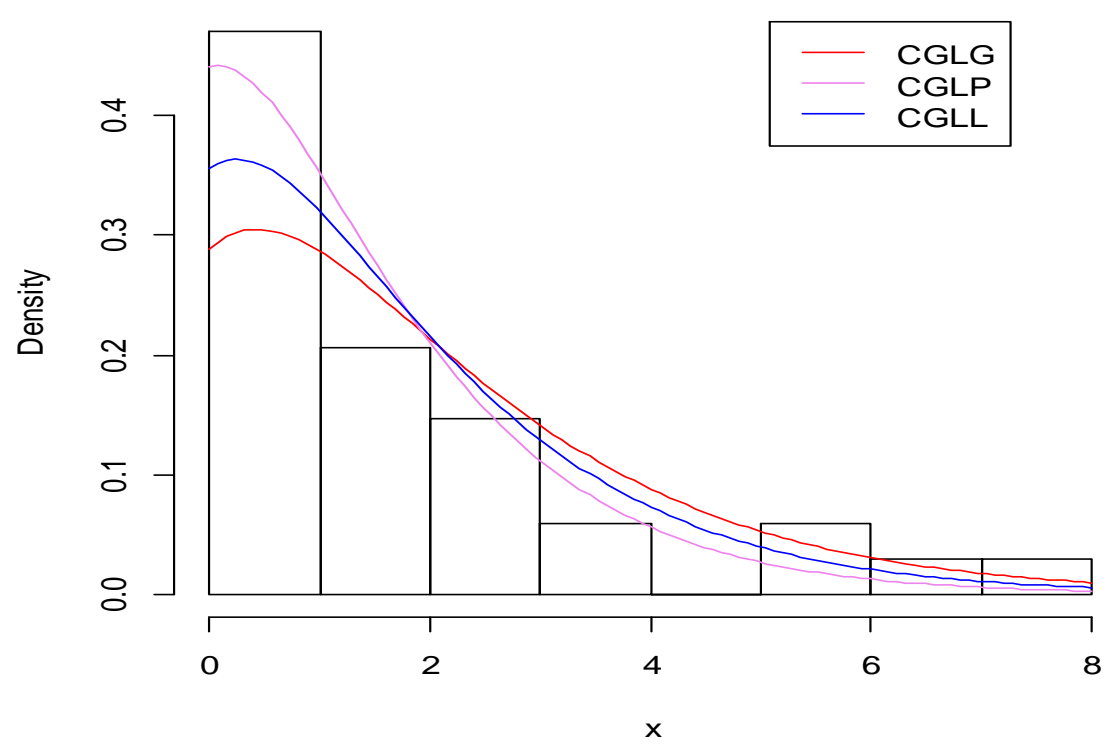

Fig 5: Fitting of CGLP, CGLG, CGLL to the data

\section{Conclusion}

We constructed a new class of compound complementary generalized lifetime distribution by compounding generalized Lindley distribution with that of power series distribution. Furthermore, we also discussed some special cases of this class of distributions that are very flexible in terms of density and hazard rate functions. Mathematical properties such as moments, order statistics and parameter estimation through MLE of the proposed class has also been discussed. Finally the potentiality of the CGLPS class has been illustrated by fitting it to model some real life data set. It is quite clear and evident from the above analysis that according to the AIC and BIC all the sub models of CGLPS family of compound distributions perform excellently well but among them generalized Lindley Poisson is the best competitor since it has lowest AIC and BIC which is also corroborated by the graphical analysis in the fig (5). Therefore, practitioners are advised to use one of our model in order to get effective results when it comes to fit lifetime data.

\section{References}

1. Adamidis, K., and Loukas, S. (1998). A lifetime distribution with decreasing failure rate.Journal of Statistics \& Probability Letters, 39, 35-42.

2. Adil, R., and Jan, T.R. (2013). A compound of zero truncated generalized negative binomial distribution with the generalized beta distribution. Journal of Reliability and Statistical Studies, 6(1), 11-19.

3. Adil, R., and Jan, T.R. (2014a). A compound of Geeta distribution with the Generalized Beta Distribution.Journal of Modern Applied Statistical Methods,13(1), 278-286. 
4. Adil, R., and Jan, T.R. (2014b). A compound of Consul distribution with the Generalized Beta Distribution. Journal of Applied probability and Statistics Methods,9(2), 17-24.

5. Adil, R., and Jan, T.R. (2015a). A new three parameter Consul Kumaraswamy distribution.International journal of Modern Mathematical Sciences, 13(4), 336376.

6. Adil, R., and Jan, T.R. (2016b).A new family of lifetime distribution with real life application.International Journal of Mathematics and Statistics, 17(1), 23-38.

7. Adil, R., Zahoor, A., and Jan, T.R. (2016a). A new count data model with application in genetics and ecology.Electronic Journal of Applied Statistical Analysis, 9(1), 213-226.

8. Adil, R., Zahoor, A., and Jan, T.R. (2016c). A new generalized class of compound lifetime distributions.AMIS, in Press

9. Barakat, H.M., and Abdelkader, Y.H.(2004).Computing the moments of order statistics from nonidentical random variables, Statistical Methods and Applications, 13, 15-26.

10. Bhaumik, D.K., Kapur, K., Gibbons, R.D.(2009). Testing parameters of a gamma distribution for small samples. Technometrics, 51, 326-334.

11. Chahkandi, M., andGanjali, M. (2009).On some lifetime distributions with decreasing failure rate, Computational Statistics \& Data Analysis, 53, 4433-4440.

12. Gerstenkorn, T. (1993). A compound of the generalized gamma distribution with the exponential one, Recherchessurlesdeformations., 16(1), 5-10.

13. Gerstenkorn, T. (1996). A compound of the Polya distribution with the beta one, RandomOper. andStoch. Equ., 4(2), 103-110.

14. Gerstenkorn, T. (2004).A compound of the generalized negative binomial distribution with the generalized beta one, CEJM. 2(4), 527-537.

15. Ghitany, M.E., Mutairi,D.K., and Nadarajah, S. (2007). Zero truncated PoissonLindley distribution and its application, Mathematics and Computers in Simulation 79 493-506.

16. Greenwood, M., and Yule, G.U. (1920).An inquiry into the nature of frequency distribution representative of multiple happenings with particular reference to the occurrence of multiple attacks of disease or of repeated accidents, J. Roy. Stat. Soc., 83, 255-279.

17. Gupta, R.C.,and Ong, S.H. (2004). A new generalization of the negative binomial distribution, Journal of Computational Statistics and Data Analysis, 45, 287-300.

18. Gurland, J. (1957). Some interrelations among compound and generalized distributions.Biometrika., 44, 265-268.

19. Kemp, A.W., and Kemp, C.D. (1966). An alternative derivation of Hermite distribution, Biometrika, 53, 627-628.

20. Kumaraswamy, P. (1980). A generalized probability density functions for doublebounded random processes. Journal of hydrology, 46(1), 79-88.

21. Li, X. H., Huang, Y. Y., and Zhao, X. Y. (2011).The Kumaraswamy Binomial Distribution.Chinese Journal of Applied Probability and Statistics, 27(5), 511521.

22. Morais, A.L., and Barreto-Souza, W. (2011).A compound class of Weibull and power seriesdistributions. Computational Statistics and Data Analysis 55, 14101425. 
23. Sankaran, M. (1970).The discrete Poisson-Lindley distribution.Biometrics, 26, 145-149.

24. Skellam, J.G. (1948). A probability distribution derived from the binomial distribution by regarding the probability of success as variable between the sets of trials, Journal of the Royal Statistical Society, Series B, 10, 257-261.

25. Tahmasbi, R., and Rezaei, S.(2008). A two-parameter lifetime distribution with decreasing failure rate,Computational Statistics \& Data Analysis, 52, 3889-3901.

26. Zakerzadeh, H., and Mahmoudi, E. (2012).A new two parameter lifetime distribution: model and properties, Arxiv.

27. Zamani, H., and Ismail, N. (2010). Negative Binomial-Lindley Distribution and Its Application. Journal of Mathematics and Statistics, 1, 4-9. 